\title{
PPARs in atherosclerosis: the clot thickens
}

\author{
Antonio Castrillo and Peter Tontonoz
}

Howard Hughes Medical Institute and Department of Pathology and Laboratory Medicine, UCLA, Los Angeles, California, USA.

\begin{abstract}
Cardiovascular disease is the leading cause of morbidity and mortality in Western countries. Previous studies have highlighted the beneficial effects of PPAR $\gamma$ activators on cardiovascular disease; however, the role of other PPAR family members in atherosclerosis is less clear. A report in this issue of the JCI expands our understanding of PPARs in vascular biology and highlights the potential use of multiple PPAR agonists to limit lipid accumulation in macrophages (see the related article beginning on page 1564).
\end{abstract}

\section{PPARs and atherosclerosis: nuclear receptor action in the artery wall}

Atherosclerosis is a chronic inflammatory disease as well as a disorder of lipid metabolism (1). The accumulation of cholesterol-rich lipoproteins in the artery wall results in the recruitment of circulating monocytes, their adhesion to the endothelium, and their differentiation into tissue macrophages. Lipidloaded macrophages play an important role in the production of chemokines, cytokines, and reactive oxygen species in the early stages of lesion formation. Therefore mechanisms that limit macrophage cholesterol accumulation and/or prevent the production of inflammatory mediators all have the potential to inhibit lesion development.

The PPAR family is comprised of 3 different proteins: PPAR $\alpha$, PPAR $\beta$ (also referred to as $\delta$ ), and PPAR $\gamma(2)$. Natural ligands for these receptors include fatty acids and oxidized fatty acids. The relevance of PPAR pathways to metabolic disease is underscored by the use of the fibrates (PPAR $\alpha$ agonists) and thiazolidinediones (PPAR $\gamma$ agonists) to treat hyperlipidemia and type 2 diabetes, respectively. The expression of PPARs in cells of the artery wall has prompted a number of investigations into the effects of PPAR agonists on atherosclerosis in mice (3). Studies on PPAR $\gamma$ are in general agreement that activation of this receptor in the artery wall is beneficial (4-6). However, studies using PPAR $\alpha$ - and PPAR $\beta$-knockout mice have yielded more complex results. Transplantation of bone

Nonstandard abbreviations used: $\mathrm{LDLR}^{-/}$, LDL receptor-deficient; LXR, liver $X$ receptor.

Conflict of interest: The authors have declared that no conflict of interest exists.

Citation for this article: J. Clin. Invest. 114:1538-1540 (2004). doi:10.1172/JCI200423705. marrow lacking PPAR $\beta$ has been reported to reduce atherosclerosis in apoE $\mathrm{E}^{-/-}$mice (7). Similarly, mice lacking both PPAR $\alpha$ and apoE were shown to develop fewer lesions (8). On the other hand, intervention studies using PPAR $\alpha$ agonists have suggested antiatherogenic effects in mice (9), and the Veterans Affairs High-Density Lipoprotein Intervention Trial showed a clear reduction in cardiovascular events in patients taking gemfibrozil (10). The impact of PPAR $\beta$ agonists on atherosclerosis is unknown, although GW1516 was shown to have beneficial effects on plasma lipid profiles in obese rhesus monkeys (11).

PPARs are known to influence pathways for both lipid uptake and efflux in macrophages. PPAR $\gamma$ promotes CD36 expression (12), and both PPAR $\alpha$ and PPAR $\gamma$ induce expression of liver $X$ receptor $\alpha(\operatorname{LXR} \alpha)$ and $\operatorname{ABCA} 1(4,13)$ (Figure $1)$. However, the ability of PPARs to control LXR $\alpha$ expression is much more prominent in human cells than in murine cells, raising the possibility that additional pathways are involved in the beneficial effects of PPARs observed in murine models. In addition to their effects on lipid metabolism, PPAR activators also inhibit inflammatory gene expression in cultured macrophages (14). Glass and colleagues have further shown that treatment of LDL receptor-deficient $\left(\mathrm{LDLR}^{-/}\right)$mice with PPAR $\gamma$ agonists reduced the expression of inflammatory mediators (5). Thus inhibition of inflammation represents a second mechanism by which PPAR activation might influence atherogenesis.

\section{Differential effects of PPAR family members on the development of atherosclerosis in mice}

In the current issue of the JCI, Li et al. (15) compare the effects of PPAR $\gamma, \operatorname{PPAR} \alpha$, and PPAR $\beta$ ligands on the development of atherosclerosis in $\mathrm{LDLR}^{-/}$mice. They observed profound atheroprotective effects of the PPAR $\alpha$ ligand GW7647, comparable to that previously observed for the PPAR $\gamma$ agonists rosiglitazone and GW7845 (5). In contrast, no change in lesion development was observed in mice treated with PPAR $\beta$ ligand. Beneficial metabolic effects of PPAR $\alpha$ ligand included reduced weight gain, reduced insulin levels, and decreased levels of VLDL and LDL fractions. No significant changes were observed with PPAR $\beta$ ligand. This shows that the ability to improve plasma lipid profiles and increase insulin sensitivity are likely to be major factors in the effects of PPAR $\alpha$ and $\gamma$ agonists on atherosclerosis observed in diabetic patients and hypercholesterolemic mice.

Li et al. (15) further explored the effects of PPAR $\alpha$ and PPAR $\beta$ agonists on gene expression in atherosclerotic mice. Each of the PPAR ligands was found to repress the expression of inflammatory markers in the artery wall even though PPAR $\beta$ did not reduce lesion formation. PPAR $\gamma$ ligand increased the expression of CD36, LXR $\alpha$, and ABCA1 and promoted cholesterol efflux in cultured macrophages. However, at the end of the study, ABCA1 expression was not induced by either ligand, and LXR $\alpha$ expression was induced only by the PPAR $\alpha$ ligand. PPAR $\gamma$ did activate LXR $\alpha$ and ABCA1 at earlier stages. Thus, neither anti-inflammatory activity nor the ability to regulate LXR correlated well with the anti-atherosclerotic effects.

\section{Effects of PPAR family members on macrophage foam-cell formation}

To further explore the mechanism of PPAR actions in atherogenesis, Li et al. (15) developed a novel approach for evaluating macrophage foam-cell formation in vivo. This approach involved the adoptive transfer of peritoneal macrophages from various PPAR null mice into the peritoneal cavity of $\mathrm{LDLR}^{-/-}$mice fed a cholesterolrich diet. This innovative assay provides a new model for macrophage lipid uptake although further validation is needed to clarify whether the assay faithfully reflects the actions of artery wall macrophages during atherogenesis. Nevertheless, Li et al. found that PPAR $\alpha$ and PPAR $\gamma$ ligands 


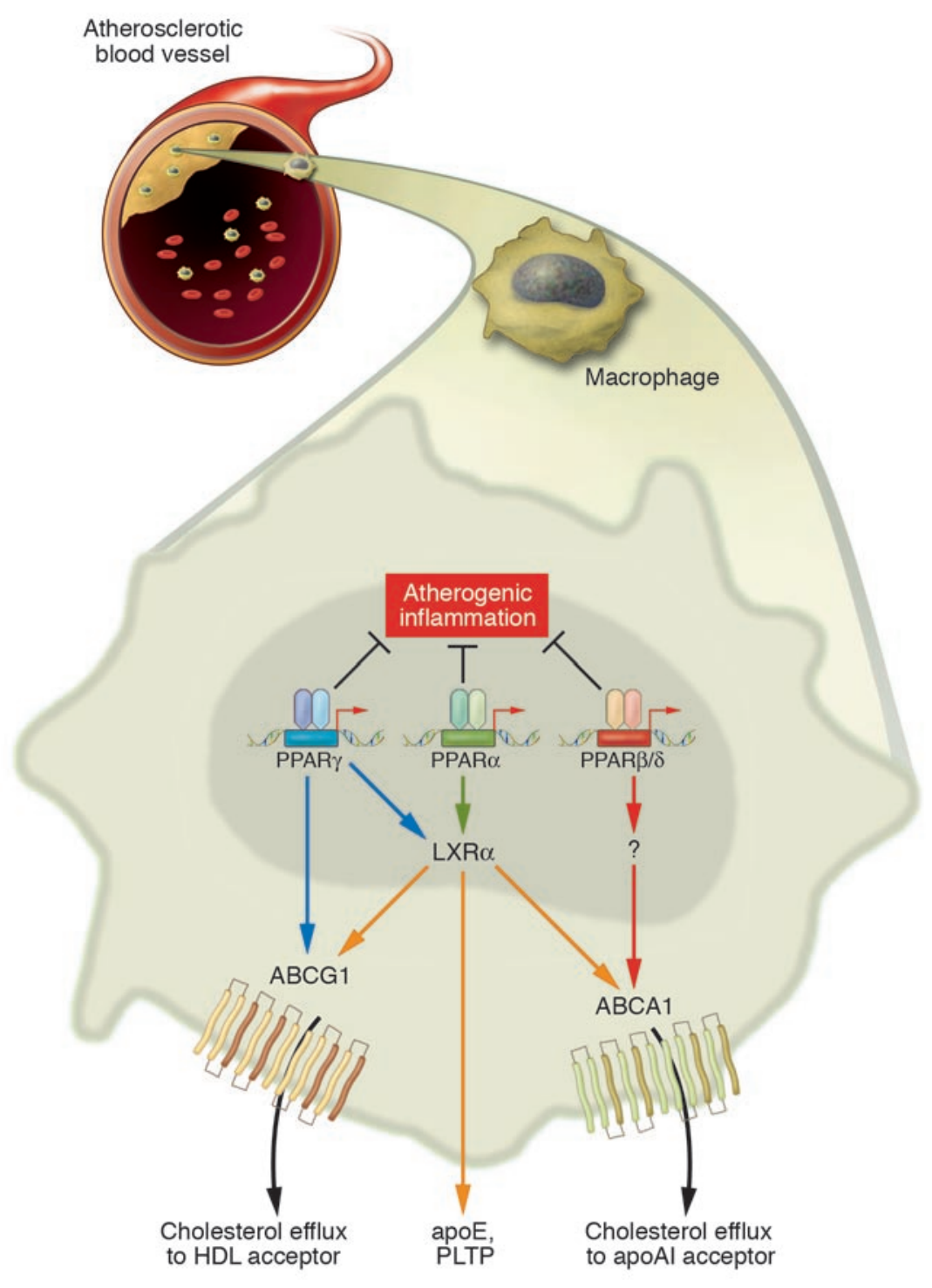

inhibited lipid accumulation in a receptor-dependent manner, pointing to direct protective mechanisms within the macrophage. PPAR $\gamma$ ligand also markedly reduced cholesterol esterification in macrophages. This newly appreciated activity may be a significant contributor to the atheroprotective effects of PPAR $\gamma$ agonists.

The authors also investigated the potential role of LXRs in the antiatherogenic effects of PPAR ligands. LXR is a key transcriptional regulator of $A B C A 1$ and ABCG1 expression and plays a major role in protection against cellular lipid overload, as deduced from studies using LXR-deficient macrophages and synthetic ligands (16-18). The results of Li et al. (15) suggest that the inhibition of foam-cell formation observed with PPAR $\alpha$ ligands requires the expression of LXRs whereas inhibition by PPAR $\gamma$ ligands does not. Interestingly, the authors found that the ability of rosiglitazone to inhibit peritoneal foam-cell formation in WT and LXR null macrophages correlated with a modest increase in ABCG1 but not ABCA1 expression. Thus the ability of PPAR $\gamma$ to promote efflux independently of LXR may involve direct effects on efflux transporters (Figure 1). ABCG1 was recently shown to increase cholesterol efflux from macrophages to HDL, but not apoAI (19) and ex vivo macrophages from PPAR $\gamma$ ligand-treated mice showed increased cholesterol efflux to HDL, consistent with this mechanism. However, the definitive role of ABCG1 in lipid metabolism and atherosclerosis in vivo has not yet been established. Furthermore, the effects of PPAR agonists on ABCG1 expression in macrophages are

\section{Figure 1}

PPAR signaling pathways influence macrophage gene expression and foam-cell formation. Ligand activation of PPAR $\alpha$ and PPAR $\gamma$, but not PPAR $\beta / \delta$, inhibits the development of atherosclerosis in $\mathrm{LDLR}^{-/}$mice. Both systemic and local mechanisms might contribute to these beneficial effects. Previous studies have suggested that PPAR $\alpha$ and PPAR $\gamma$ increase $L X R \alpha$ expression in macrophages and promote expression of ABCA1, which mediates cholesterol efflux to apoAI. Results from the study in this issue by Li et al. (15) suggest that PPAR $\gamma$ may also inhibit cholesterol accumulation in macrophages through direct regulation of ABCG1, which has been implicated in cholesterol efflux to HDL. Activation of each of the PPARs with selective agonists also inhibits the expression of inflammatory markers in the artery wall. These findings reinforce potential use of PPAR agonists as antiatherosclerotic therapies. PTLP, phospholipid transfer protein.

small compared to those of LXR agonists (18), and the possibility that ABCG1 might be a direct target of PPAR $\gamma$ remains to be explored. In the future, it will be of interest to determine whether the atheroprotective effects of PPAR agonists are lost in mice lacking ABCG1.

\section{Conclusions}

The study by Li et al. (15) provides new insights into pathways regulating macrophage lipid accumulation and rounds out the family picture of PPARs in atherosclerosis (Figure 1). Both PPAR $\alpha$ and PPAR $\gamma$ ligands were shown to protect against atherosclerosis in $\mathrm{LDLR}^{-/-}$mice and inhibit macrophage foam-cell formation. In contrast, the authors did not observe any effect from PPAR $\beta$ activation. Given the discrepancies between PPAR $\beta$ agonist effects in mice and primates, however, the possibility that PPAR $\beta$ ligands may have beneficial effects on cardiovascular disease in humans is not excluded by the present study. Finally the work of Li et al. emphasizes that PPAR agonists are likely to exert their antiatherosclerotic properties by multiple mechanisms, including improving systemic lipid levels, improving insulin resistance, and inhibiting the accumulation of macrophage foam cells.

Address correspondence to: Peter Tontonoz, Howard Hughes Medical Institute, UCLA, Box 951662, Los Angeles 900951662, California, USA. Phone: (310) 206-4546; Fax: (310) 267-0382; E-mail: ptontonoz@mednet.ucla.edu. 
1. Lusis, A.J. 2000. Atherosclerosis. Nature. 407:233-241. 2. Castrillo, A., and Tontonoz, P. 2004. Nuclear receptors in macrophage biology: at the crossroads of lipid metabolism and inflammation. Annu. Rev. Cell Dev. Biol. 20:455-480.

3. Marx, N., Duez, H., Fruchart, J.C., and Staels, B 2004. Peroxisome proliferator-activated receptors and atherogenesis: regulators of gene expression in vascular cells. Circ. Res. 94:1168-1178.

4. Chawla, A., et al. 2001. A PPARgamma-LXR-ABCA1 pathway in macrophages is involved in cholesterol efflux and atherogenesis. Mol. Cell. 7:161-171.

5. Li, A.C., et al. 2000. Peroxisome proliferator-activated receptor $\gamma$ ligands inhibit development of atherosclerosis in LDL receptor-deficient mice. J. Clin. Invest. 106:523-531.

6. Collins, A.R., et al. 2001. Troglitazone inhibits formation of early atherosclerotic lesions in diabetic and nondiabetic low density lipoprotein receptor-deficient mice. Arterioscler. Thromb. Vasc. Biol. 21:365-371.

7. Lee, C.H., et al. 2003. Transcriptional repression of atherogenic inflammation: modulation by PPARdelta. Science. 302:453-457.
8. Tordjman, K., et al. 2001. PPAR $\alpha$ deficiency reduces insulin resistance and atherosclerosis in apoE-null mice. J. Clin. Invest. 107:1025-1034.

9. Duez, H., et al. 2002. Reduction of atherosclerosis by the peroxisome proliferator-activated receptor alpha agonist fenofibrate in mice. J. Biol. Chem. 277:48051-48057.

10. Rubins, H.B., et al. 1999. Gemfibrozil for the secondary prevention of coronary heart disease in men with low levels of high-density lipoprotein cholesterol. Veterans Affairs High-Density Lipoprotein Cholesterol Intervention Trial Study Group. N. Engl. J. Med. 341:410-418.

11. Oliver, W.R., Jr., et al. 2001. A selective peroxisome proliferator-activated receptor delta agonist promotes reverse cholesterol transport. Proc. Natl. Acad. Sci. U. S. A. 98:5306-5311.

12. Tontonoz, P., Nagy, L., Alvarez, J.G., Thomazy, V.A. and Evans, R.M. 1998. PPARgamma promotes monocyte/macrophage differentiation and uptake of oxidized LDL. Cell. 93:241-252.

13. Chinetti, G., et al. 2001. PPAR-alpha and PPARgamma activators induce cholesterol removal from human macrophage foam cells through stimula- tion of the ABCA1 pathway. Nat. Med. 7:53-58.

14. Ricote, M., Li, A.C., Willson, T.M., Kelly, C.J., and Glass, C.K. 1998. The peroxisome proliferator-activated receptor-gamma is a negative regulator of macrophage activation. Nature. 391:79-82.

15. Li, A.C., et al. 2004. Differential inhibition of macrophage foam-cell formation and atherosclerosis in mice by PPAR $\alpha, \beta / \delta$, and $\gamma . J$. Clin. Invest. 114:1564-1576. doi:10.1172/JCI200418730.

16. Joseph, S.B., et al. 2002. Synthetic LXR ligand inhibits the development of atherosclerosis in mice. Proc. Natl. Acad. Sci. U. S. A. 99:7604-7609.

17. Tangirala, R.K., et al. 2002. Identification of macrophage liver $\mathrm{X}$ receptors as inhibitors of atherosclerosis. Proc. Natl. Acad. Sci. U. S. A. 99:11896-11901.

18. Venkateswaran, A., et al. 2000. Human white/ murine ABC8 mRNA levels are highly induced in lipid-loaded macrophages. J. Biol. Chem. 275:14700-14707.

19. Wang, N., Lan, D., Chen, W., Matsuura, F., and Tall, A.R. 2004. ATP-binding cassette transporters G1 and G4 mediate cellular cholesterol efflux to highdensity lipoproteins. Proc. Natl. Acad. Sci. U. S. A. 101:9774-9779.

\title{
Fusion of bone marrow-derived stem cells with striated muscle may not be sufficient to activate muscle genes
}

\author{
Giulio Cossu
}

Stem Cell Research Institute, DIBIT, Milan, Italy.

\begin{abstract}
Several studies have demonstrated the existence of pluripotent bone marrow-derived stem cells capable of homing to injured cardiac and skeletal muscle; however, there has been little evidence demonstrating the induction of tissue-specific endogenous genes in donor stem cells following engraftment. A new study in this issue reports an intriguing finding that raises additional concerns relating to stem cell plasticity and stem cell therapy in an already heated and controversial field. The study demonstrates that wild-type bone marrow-derived side population stem cells are indeed readily incorporated into both skeletal and cardiac muscle when transplanted into mice that lack $\delta$-sarcoglycan - a model of cardiomyopathy and muscular dystrophy. However, these cells fail to express sarcoglycan and thus to repair the tissue, which suggests that this stem cell population has limited potential for cardiac and skeletal muscle regeneration (see the related article beginning on page 1577).
\end{abstract}

Bone marrow-derived side population (BM-SP) stem cells have the ability to repopulate the hematopoietic system (1) and to colonize, at low frequencies, many different tissues, including skeletal (2) and cardiac muscle (3). The $\delta$-sarcoglycan-null

Nonstandard abbreviations used: BM-SP, bone marrow-derived side population.

Conflict of interest: The author has declared that no conflict of interest exists.

Citation for this article: J. Clin. Invest. 114:1540-1543 (2004). doi:10.1172/JCI200423733. mouse is a model of cardiomyopathy and muscular dystrophy (corresponding to a human limb-girdle myopathy) and develops microinfarcts in heart and skeletal muscle (4). Muscle cell death in this model is related to the lack of $\delta$-sarcoglycan, which causes loss of the other sarcoglycans $(\alpha, \beta$, and $\gamma)$ and in turn disassembly of the dystroglycan complex (Figure 1), which is composed of several proteins that link the cytoskeleton to the basal lamina and reduce membrane stress during contraction. Absence or reduction of the dystroglycan complex results in increased fragility of the membrane and increased chance of damage to the muscle cell. Resident cells capable of repairing injured skeletal muscle (satellite cells) are well characterized (5), and despite the long-held belief that heart cells cannot regenerate, evidence for the existence of cells with a similar reparative function in the heart has only begun to accumulate during the last 2 years $(6,7)$. This observation suggests that injured $\delta$-sarcoglycan-deficient tissues should recruit both local and blood-born stem cells that may contribute to regeneration.

In this issue of the JCI, Lapidos et al. (8) transplanted BM-SP stem cells from wild-type male mice into female $\delta$-sarcoglycan-null mice, and their results, consistent with those from previous studies of stem cell-mediated skeletal and cardiac muscle regeneration, demonstrated the presence of these donor cells, determined by the presence of a $\mathrm{Y}$ chromosome inside cardiac and skeletal muscle at the expected frequency. A fraction of the nuclei of these Y chromosome-positive donor cells was unequivocally shown to be present inside the cytoplasm of several differentiated car- 